\title{
Limitations of a dynamic shear-frame model based in a small-scale experimental steel structure
}

\author{
Augusto de S. Pippi ${ }^{1, *}$, Pedro L. Bernardes Júnior ${ }^{2}$, Suzana M. Avila ${ }^{2}$, Marcus V. G. de \\ Morais $^{2}$, and Graciela Doz ${ }^{1}$ \\ ${ }^{1}$ PECC, Postgraduate program in Structural Engineering and Construction, University of Brasilia \\ (UnB), Campus Darcy Ribeiro, 70910-900, Brasilia, Brazil. \\ ${ }^{2} \mathrm{PPG}$, Postgraduate program in Engineering Material Integrity, University of Brasilia (UnB), Campus \\ of Gama (FGA), 72444-240, Gama (Brasilia), Brazil.
}

\begin{abstract}
Many engineering problems require geometric modeling and mechanical simulation of structures. Through the structural models, engineers try to simulate the real behavior of these structures. It is important that a model contain all the necessary parameters that describe the structure and its behavior during its useful life. In the field of dynamics, one of the most used models is the shear-frame, in which the stiffness of the structure is given by the stiffness of the columns and the whole mass is concentrated in the floor levels, which are considered with infinite stiffness. In some cases, this simplification offers more conservative results, which can lead to considerable errors, especially in the case of natural frequencies. Knowing that the quality of a structural model depends on the simplifications considered, an experimental 3D steel frame, constructed to typify the dynamic behavior of a tall building, was tested with a data acquisition system and accelerometers, in order to obtain its natural frequencies. In addition, a numerical model was developed in order to ascertain the results. These values of natural frequencies are compared with an idealized shear-frame model obtained from the experimental model. This comparison allows a critical analysis of the numerical models that can be employed to represent the real dynamic behavior of structures. The aim of the investigation is to show the results of the modal analysis for each model, comparing them with the experimental results and commenting their advantages and the limitations.
\end{abstract}

\section{Introduction}

With the advent of new materials and technologies, the sturdy and heavy constructions gave way to lighter and slimmer structural systems. As a result, current buildings are generally affected by effects that can cause human discomfort, and this is now a considerable problem in buildings $[1,2]$.

\footnotetext{
${ }^{*}$ Corresponding author: augustopippi@hotmail.com
} 
When the dynamic properties of a structure are unknown, it can be severely affected by actions such as earthquakes and strong winds. Before the natural frequencies of a building can be computed, an idealized theoretical model of the structure must be constructed. This theoretical idealization may be responsible for major errors in the result. The reason for inadequate idealizations is that buildings are complicated structures and there is only a limited understanding of how they actually behave. [3]. Thus, the dynamic properties of buildings are often not estimated properly.

Many studies are carried out in order to measure properties in already built buildings or to estimate parameters necessary to obtain these properties numerically, such as stiffness and mass distribution in the structure. [3-11]. In most of these studies, a numericaltheoretical model is constructed in order to verify the results obtained. However, a divergence between the experimental results and the numerically obtained values is observed. Thus, more realistic structural models are needed to ensure the behavior of the constructed structure.

In this sense, a small-scale experimental model of a building was tested and values of natural frequencies and modes of vibration were computed. Two idealized numerical models, one in finite elements using the SAP $2000^{\circledR}$ software and another using the classic shear-frame approximation, were analyzed in order to ascertain the results obtained experimentally. This comparison allows a critical analysis of the numerical models that can be used to represent the most realistic dynamic behavior of the structures.

\section{Shear-frame model}

The shear-frame model is often used in the study of the response to lateral forces of frame structures subjected to dynamic loading. It is known by its simplicity of the equilibrium equations, which are easily solved by computer methods. In this model the whole mass of the floor is concentrated in the direction of the corresponding lateral degrees of freedom. In traditional shear-frame models, the rotation of the nodes is considered equals to zero, which corresponds to the beams having infinite stiffness when compared to the stiffness of the columns. [12, 13].

Although it is a model of easy manipulation, it is known to provide results that can be very different from the real behavior of the structural system, besides not considering the effect of the axial deformations on the column flexural stiffness. Hurty and Rubinstein [14] showed in their studies that when considering nodes rotation the difference in natural frequencies values, regarding the unbending floor, can reach 109\%. Discrepancies may occur when considering shear-frame modeling. Although this approach can predict natural frequencies with some accuracy, it does not lead to identical results comparing to real deformation.[13, 15].

Johnson [16] did an analysis of a plane frame with a single floor through an analytical model considering node rotation and compared with a shear-frame model. The fundamental frequency difference between the two models was $25 \%$. Another consideration in the traditional shear-frame model is to consider the basis of the set structure embedded.

In this sense Ward [5] showed that the constraint set at the base is an important feature when talking about dynamic response of buildings. 


\section{Analysis}

\subsection{Experimental analysis}

To simulate the behavior of a building experimentally, a reduced model was constructed [17]. This model consists of ten SAE 1020 steel modules, with Young's modulus of $205 \mathrm{GPa}$ and a density of $7870 \mathrm{~kg} / \mathrm{m}^{3}$. Each module is composed of two plates connected to each other by four $200 \mathrm{~mm}$ high columns. All plates and columns have a thickness of $6.3 \mathrm{~mm}$ and dimensions as shown in Figure 1.
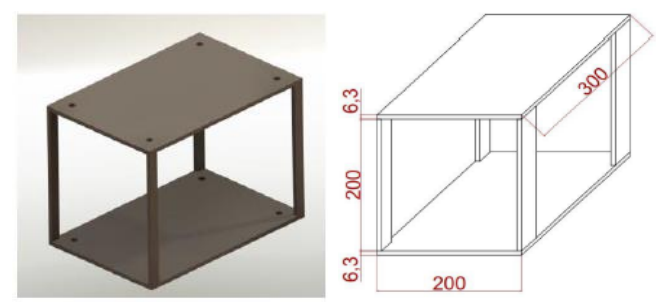

Fig. 1. Dimension of module SAE 1020.

Although the theoretical dimensions of the module elements are the same for each one of them, small variations are known to occur during the construction process. Thus, for all modules, the dimensions of the plates, columns and holes were measured. In addition, all bolts and nuts used were weighed and separated so that they were always used in the same positions and at the same levels.

For the assembly of the model, shown in Figure 2, the first module was attached to fixed base in the Vibration Laboratory of the University of Brasilia and the following modules were connected, one by one, resulting in a structure approximately $2.12 \mathrm{~m}$ high and with approximate slenderness of 1:10.

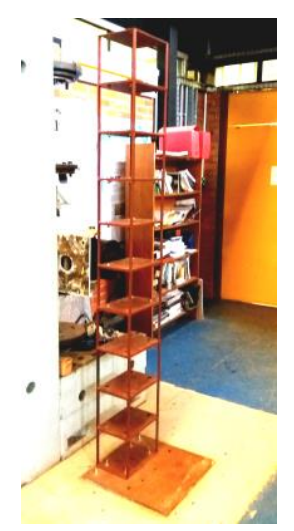

Fig. 2. Experimental model.

In order to obtain the natural frequencies of the experimental model, two tests of free vibration, one in each direction, were carried out with an impact hammer and two accelerometers: one for reference and other for measuring. Both accelerometers are made of quartz and have a sensitivity of $10 \mathrm{mV} / \mathrm{g}$. Thus, the structure was excited with an impulse always in the same place, keeping also fixed the position of the reference accelerometer. The measurement accelerometer was positioned at three different points of the structure: at both ends and at the center of the last level of the frame, as shown in Figure 3. 
(a)

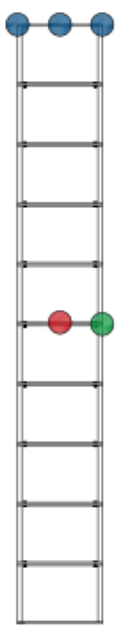

MEASURE

IMPULSE

REFERENCE

(b)

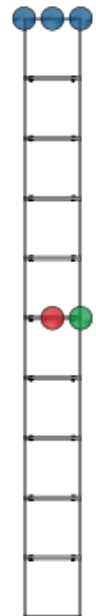

Fig. 3. Positioning diagram of accelerometers and impulse in the test for the direction of lower inertia (a) and greater inertia (b).

\subsection{Numerical analysis}

From the geometry and material properties of the experimental frame, a finite element model (FEM) was constructed in SAP $2000^{\circledR}$ software. The modeling of the columns used the frame element and the floors were modeled with the equivalent grid slab, also using frame element. To simulate the four screws that attach the frame to a fixed plate on the floor basis, spring elements were used. The finite element model is shown in Figure 4. In order to replicate the same experimental natural frequency attainment, a harmonic analysis was performed applying a force at the same location as the experimental test, thus simulating the hammer impulse.
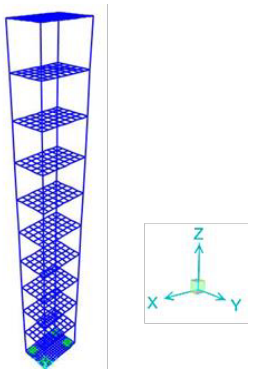

Fig. 4. Finite element model of experimental structure.

For a shear-frame model of the experimental structure, the mass of each floor was considered as the total mass of the plates, screws and nuts, subtracting the mass of the holes. The stiffness of each floor was assumed to be the sum of the stiffness of all the columns of the floor, in the direction considered, $K_{n}(\mathrm{~N} / \mathrm{m})=12 E I / L_{n}{ }^{3}$, where, $E$ is the Young's modulus, $I$ is the moment of inertia of the cross-section and $L_{n}$ is the length of the column. The columns were considered as fixed at both ends, due to the welds between the columns and the plates. According to the classic shear-frame model, the base is considered fixed, the mass of the columns is disregarded, which is according to the bibliography, and the floor considered with infinite stiffness, which prevents rotation. 


\section{Results}

An experimental modal analysis was performed in the reduced model to obtain frequencies and modes. The first three modes of vibration were translation in the direction of lower stiffness, translation in the direction of greater stiffness and torsion, respectively. For the two numerical models, finite elements tridimensional model and shear-frame, a free vibration analysis was performed to obtain corresponding frequencies and vibration modes. In the analysis by finite elements, the first three modes of vibration agreed with those obtained in the experimental analysis. The natural frequencies obtained experimentally and through the numerical analysis of the two models are presented in Table 1 below.

Table 1. Natural Frequencies.

\begin{tabular}{c|ccc}
\hline \multirow{2}{*}{$\begin{array}{c}\text { Vibration } \\
\text { Mode }\end{array}$} & \multicolumn{3}{|c}{ Natural Frequency $(\mathrm{Hz})$} \\
\cline { 2 - 4 } & Experimental & FEM & Shear-frame \\
\hline $1^{\circ}$ & 4.01 & 4.16 & 7.35 \\
$2^{\mathrm{o}}$ & 6.85 & 6.65 & - \\
$3^{\mathrm{o}}$ & 12.15 & 12.98 & - \\
\hline
\end{tabular}

It can be noticed that the numerical model by finite elements was efficient representing experimental results. The shear-frame model has only lateral modes and its fundamental frequency has a difference of almost $83 \%$ compared to the first experimental frequency.

\section{Conclusions}

A comparison between experimental, numerical tridimensional model and the shear-frame model was performed in this work. The results showed that the shear-frame model was not efficient to represent the behavior of the experimental structure. This is possibly due to the simplifications imposed by the model, for example, disregarding the rotation of the nodes and the base of the structure is considered to be fixed. Another factor that may be important is that the shear-frame model cannot predict, because it is two-dimensional, the torsional modes, which can be very relevant in the analysis of buildings. [18, 19]. Simplified models therefore need to be used carefully and comparisons of calculated values with experimental values may show discrepancies unless much care is taken with modeling which may entail the inclusion of the effect of stiffness of non-structural elements, soil-structure interaction, conditions of linkages between elements, among other factors $[3,14,16]$.

The authors gratefully acknowledge the Brazilian agency CNPq (Council of National Scientific and Technological Development) for financial support of this study.

\section{References}

[1] K.S. Moon, Dynamic Interrelationship between Technology and Architecture in Tall Buildings (Massachusetts Institute of Technology, Cambridge, 2005)

[2] M.M. Ali, K.S. Moon, Archit. Sci. Rev. 49.2, pp. 179-182. (2007)

[3] B. Ellis, Proc. Inst. Civ. Eng., 69, 3, pp. 763-776. (1980)

[4] R. Crawford, H.S. Ward, Bull. Seismol. Soc. Am., 54, pp. 1743-1756. (1964)

[5] H.S. Ward, Proceedings Institution of Civil Engineers, 43, pp. 553-572. (1969) 
[6] A. Schultz, 118, 1, pp. 243-263. (1992)

[7] J.Y. Kim, D.Y. Kim, S.D. Kim, CTBUH 8th World Cong., pp. 1-7. (2008)

[8] K.C.S. Kwok, K.T. Tse, S. Campbell, Adv. Struct. Eng., 14, 6, pp. 1107-1128. (2011)

[9] J.T. Snaebjornsson, E.R. Thorhallsson, 22nd Czech Conc. Day, pp. 79-108. (2015)

[10] G. Acunzo, N. Fiorini, F. Mori, D. Spina, Mech. Syst. Signal Process., 98, pp. 580593. (2018)

[11] H.P. Lin, S.C. Chang, C. Chu, J. Mech., 32, 5, pp. 501-514. (2016)

[12] G.C. Ezeokpube, N.N. Osadebe, Niger. J. Technol., 29, 1, pp. 5-12. (2010)

[13] E.C. Mbanusi, D.A. Obodoh, Int. J. Eng. Comput. Sci., 5, 5, pp. 16620-16638. (2016)

[14] W.C. Hurty, M.F. Rubinstein, Dynamics of Structures (Prentice-Hall of India Private Limited, New Delhi, 1967)

[15] R.W. Clough, J. Penzien, Dynamics of Structures (Computers \& Structures, Berkeley, 2003)

[16] D. Johnson, Advanced Structural Mechanics (D. Johnson and Thomas Telford Limited, London, 2000)

[17] P.L. Bernardes Junior, G.B. Colheirinhas, M.V.G. Morais, S.M. Avila, Proceedings of XXXVIII Ibero-Latin American Congress on Computational Methods in Engineering, p. 14. (2017)

[18] V. Gupta, P.S. Pajgade, Int. J. Innov. Res. Sci. Eng. Technol., 1, 8, pp. 168-173. (2015)

[19] R. Thaskeen, S. Shajee, Int. J. Innov. Res. Sci. Eng. Technol., 5, 9, pp. 18861-18871. (2016) 